\title{
Inverse proximity effect in semiconductor Majorana nanowires
}

\author{
Alexander A. Kopasov*1, Ivan M. Khaymovich ${ }^{1,2}$ and Alexander S. Mel'nikov ${ }^{1,3}$
}

\section{Full Research Paper}

Address:

${ }^{1}$ Institute for Physics of Microstructures, Russian Academy of Sciences, 603950 Nizhny Novgorod, GSP-105, Russia, ${ }^{2}$ Max Planck Institute for the Physics of Complex Systems, 01187 Dresden, Germany and ${ }^{3}$ Lobachevsky State University of Nizhny Novgorod, 23 Gagarina, 603950 Nizhny Novgorod, Russia

Email:

Alexander A. Kopasov* - kopasov@ipmras.ru

* Corresponding author

Keywords:

inverse proximity effect; Majorana fermions; semiconducting nanowires

\section{Open Access}

Beilstein J. Nanotechnol. 2018, 9, 1184-1193.

doi:10.3762/bjnano.9.109

Received: 30 November 2017

Accepted: 09 March 2018

Published: 16 April 2018

This article is part of the Thematic Series "Topological materials".

Guest Editor: J. J. Palacios

(C) 2018 Kopasov et al.; licensee Beilstein-Institut. License and terms: see end of document.

\begin{abstract}
We study the influence of the inverse proximity effect on the superconductivity nucleation in hybrid structures consisting of semiconducting nanowires placed in contact with a thin superconducting film and discuss the resulting restrictions on the operation of Majorana-based devices. A strong paramagnetic effect for electrons entering the semiconductor together with spin-orbit coupling and van Hove singularities in the electronic density of states in the wire are responsible for the suppression of superconducting correlations in the low-field domain and for the reentrant superconductivity at high magnetic fields in the topologically nontrivial regime. The growth of the critical temperature in the latter case continues up to the upper critical field destroying the pairing inside the superconducting film due to either orbital or paramagnetic mechanism. The suppression of the homogeneous superconducting state near the boundary between the topological and non-topological regimes provides the conditions favorable for the Fulde-Ferrel-Larkin-Ovchinnikov instability.
\end{abstract}

\section{Introduction}

The transport phenomena in semiconducting wires with induced superconducting ordering and strong spin-orbit interaction are in the focus of current experimental and theoretical research in field of nanophysics and quantum computing [1-10]. The interest in these systems is stimulated by the perspectives of their use for design of topologically protected quantum bits. The key idea is based on the observation that for a certain range of parameters and rather strong applied magnetic fields $H$ the induced superconducting order parameter reveals so called p-wave symmetry realizing, thus, a model of Kitaev's chain [1].
The edges of such wires can host the subgap quasiparticle states that are considered as a realization of Majorana particles in condensed matter systems [11-16].

In most cases, theoretical studies of these Majorana wires are based on a simplified model of the superconducting correlations described by a phenomenological gap potential inside the wire $[3,4]$ placed in contact with a standard s-wave superconductor (Figure 1). This model, while being useful in many cases for a qualitative understanding of the induced superconduc- 
tivity, is known to possess still a number of important shortcomings. An obvious way to overcome these shortcomings is to use the microscopic theory of the proximity effect [17-24], i.e., Gor'kov equations. The microscopic approach allows one to get the effective gap operator analogous to the one used in the phenomenological model. On top of that it gives the gap dependence on the transparency of the interface between the wire and the s-wave superconductor and chemical potential via density of states (DOS). Another important point is that the exchange of electrons between the wire and superconductor can cause a so-called inverse proximity effect, i.e., the suppression of the gap function at the superconductor surface. For a rather thin superconducting shell covering the wire this gap suppression can result in the change of the superconducting critical temperature of the whole system. The analysis of this inverse proximity phenomenon is important to find out the optimal range of parameters that allows one to realize the switching between the topologically trivial and nontrivial states of the semiconducting wire used in various braiding protocols.

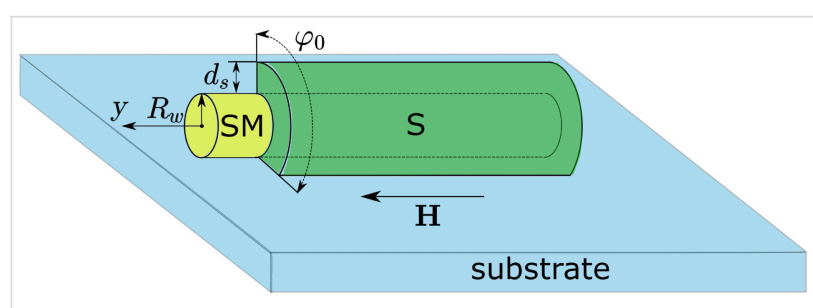

Figure 1: Schematic picture of the semiconducting wire (yellow) covered by the superconducting layer (green) placed on a substrate (light blue). $R_{w}, d_{s}$ and $\varphi_{0}$ show linear and azimuthal dimensions. The magnetic field $H$ is applied along the wire axis $O y$ while the Rashba spin-orbit vector is perpendicular to the substrate (not shown).

The goal of this work is the self-consistent analysis of the critical-temperature behavior of the wires while considering the influence of the inverse proximity effect on the induced superconducting ordering. For this purpose we start from the full set of microscopic equations for the Green functions taking into account both scattering rates describing the quasiparticle transfer between the superconducting film and the wire [17] The first rate, $\gamma_{s}$, characterizes the electron leakage from the wire to the superconductor and is responsible for the energylevel broadening in the wire. The second rate, $\gamma_{w}$, corresponds to the reverse process. These rates are determined both by the probability of electron tunneling through the barrier at the superconductor/semiconductor (S/SM) interface and the corresponding densities of states. In particular, it is important that the rate $\gamma_{w}$ is proportional to the DOS in the SM nanowire resulting in its non-trivial energy dependence. Indeed, considering, e.g., a single-channel nanowire we get the DOS diverging as a square root function of the energy relative to the bottom of the conduction band. This van Hove singularity in the DOS should cause a strong energy dependence of the scattering rate $\gamma_{w}$ and, as a consequence, the superconducting critical temperature should depend on the position of the Fermi level with respect to the bottom of the one-dimensional conduction band in the SM wire. The influence of the van Hove singularity on superconductivity should be also accompanied by the strengthening of the paramagnetic effect. Indeed, one can naturally expect that the scattering rate $\gamma_{w}$ could result in an additional effective Zeeman field induced in the superconductor due to the electron exchange with the SM wire. Due to the divergence in the DOS together with the large $g$-factor in the wire this induced Zeeman field can even exceed the value of the usual Zeeman field. Under such conditions the field dependence of the critical temperature would have a minimum near the fields $H \approx\left|\mu_{w}\right| / g \beta$, where $\mu_{w}$ is the Fermi energy of the wire relative to the bottom of its conduction band at $H=0$ and $\beta$ is the Bohr magneton. Strictly speaking, the spin-orbit interaction may cause the emergence of the third van Hove singularity below $-g \beta H / 2$, but it appears only at rather large spin-orbit interaction strengths. Note that for a vanishing induced superconducting gap $\Delta_{\text {ind }}$ this field separates the regimes with trivial and nontrivial topological properties of the system $[3,4,18]$. Further increase in the magnetic field is known to suppress the proximity effect since in the absence of the spin-orbit coupling the Fermi level crosses the only energy branch with a complete spin polarization along the magnetic field direction. The nonzero spin-orbit coupling destroys this spin polarization mixing different spin projections and resulting in a nonzero induced superconducting gap in the wire of approximately $\alpha \Delta_{\text {ind }} / g \beta H$, where $\Delta_{\text {ind }}$ is the induced superconducting order parameter in the wire, and $\alpha$ is the spin-orbit coupling constant. Still, even in the presence of the spin-orbit coupling the increasing magnetic field suppresses the induced superconductivity, which definitely restores the superconducting order parameter in the S film. This reentrant superconductivity stimulated by the magnetic field can only be maintained up to the upper critical field associated with either orbital or intrinsic paramagnetic effect in the S shell.

The suppression of the superconducting order parameter near the line of transition between the topologically trivial and nontrivial phases can result in one more interesting phenomenon: Similarly to the standard paramagnetic effect this suppression can cause the transition into the analogue of the so-called Fulde-Ferrel-Larkin-Ovchinnikov (FFLO) [25,26] state with the spatially modulated superconducting order parameter.

The paper is organized as follows: In section "Basic equations" we give the main equation of our model. Section "Results and Discussion" is devoted to the description of the solution and the analysis of the phase diagrams. In the Conclusion section we summarize our results and the suggestions for the experiment. 


\section{Basic Equations}

Hereafter we consider a long 1D semiconducting wire partially covered by a thin superconducting shell with the thickness $d_{s} \ll \xi_{s}$, where $\xi_{s}$ is the superconducting coherence length. In the cross section of the wire the superconducting film covers the angular sector $\varphi_{0}$. The model system is schematically shown in Figure 1. Hereafter we use the units with $k_{\mathrm{B}}=\hbar=1$, where $k_{\mathrm{B}}$ is the Boltzmann constant, and $\hbar$ is the Planck constant. The Hamiltonian of the system reads:

$$
\mathcal{H}=\mathcal{H}_{s}+\mathcal{H}_{w}+\mathcal{H}_{t}
$$

with the first term

$$
\begin{aligned}
\mathcal{H}_{s} & =d_{s} R_{w} \int \mathrm{d} y \mathrm{~d} \varphi\left[\psi_{\sigma}^{\dagger}(\mathbf{r}) \varepsilon_{s}(\mathbf{r}) \psi_{\sigma}(\mathbf{r})\right. \\
& \left.+\Delta_{s}(\mathbf{r}) \psi_{\uparrow}^{\dagger}(\mathbf{r}) \psi_{\downarrow}^{\dagger}(\mathbf{r})+\Delta_{s}^{*}(\mathbf{r}) \psi_{\downarrow}(\mathbf{r}) \psi_{\uparrow}(\mathbf{r})\right],
\end{aligned}
$$

describing the s-wave superconducting shell.

$$
\mathcal{H}_{w}=S_{w} \int \mathrm{d} y a_{\sigma}^{\dagger}(y)\left[\varepsilon_{w}(y)-i \alpha \hat{\sigma}_{x} \partial_{y}+h \hat{\sigma}_{y}\right]_{\sigma \sigma^{\prime}} a_{\sigma^{\prime}}(y)
$$

corresponds to the Hamiltonian of the nanowire, and the tunnel Hamiltonian takes the form

$$
\begin{aligned}
\mathcal{H}_{t}= & \sqrt{d_{s} R_{w} S_{w}} \int \mathrm{d} y \mathrm{~d} \varphi\left[\psi_{\sigma}^{\dagger}(\varphi, y) \mathcal{T}(\varphi, y) a_{\sigma}(y)\right. \\
& \left.+a_{\sigma}^{\dagger}(y) \mathcal{T}^{\dagger}(\varphi, y) \psi_{\sigma}(\varphi, y)\right] .
\end{aligned}
$$

Here $\sigma=\uparrow, \downarrow$ denotes spin degrees of freedom (summation over repeated spin indices is always assumed throughout the paper), while $\hat{\sigma}_{m}(m=x, y, z)$ are the Pauli matrices in the spin space. $R_{w}$ is the radius and $S_{w}=\pi R_{w}^{2}$ is the cross-sectional area of the wire, $(\mathbf{r})=\left(R_{w}, \varphi, y\right), \varphi$ is the polar angle in the plane perpendicular to the wire axis, which changes in the interval $0<\varphi<\varphi_{0} . y$ denotes the coordinate along the wire, $\varepsilon_{s}(\mathbf{r})=-\nabla_{\mathbf{r}}^{2} / 2 m_{s}-\mu_{s}$ and $\varepsilon_{w}(y)=-\partial_{y}^{2} / 2 m_{w}-\mu_{w}$ stand for the quasiparticle kinetic energies in the shell and in the wire with respect to the corresponding chemical potentials $\mu_{s}$ and $\mu_{w}$. $m_{s}$ and $m_{w}$ are the effective masses of the electrons in the subsystems, $\Delta_{S}(\mathbf{r})$ is the superconducting order parameter, $\alpha$ is the spin-orbit coupling constant, $h=g \beta H / 2$ is the Zeeman energy, and $H$ is the applied magnetic field.

We consider the incoherent tunneling model, which does not conserve the momentum, e.g., due to the presence of the disorder at the interface. Thus, the ensemble average of the tunneling amplitudes has the form:

$$
\overline{\mathcal{T}(\mathbf{r}) \mathcal{T}\left(\mathbf{r}^{\prime}\right)}=t^{2} \ell_{c} \delta\left(y-y^{\prime}\right) \delta\left(\varphi-\varphi^{\prime}\right)
$$

where $\ell_{c}$ is the length of the order of the atomic scale. The tunneling is also assumed to be independent of energy and spin and occurs locally in time and in space, i.e., from a point $\mathbf{r}$ on the superconducting shell into the point $y$ in the wire and back with the amplitude $\mathcal{T}(\mathbf{r})$.

It is important to note that here we do not consider the orbital effects in the superconducting shell. This approximation imposes some restrictions on the value of magnetic fields under consideration, which are nevertheless quite realistic for the experiments aimed at the manipulation with Majorana states in such systems. It is the large $g$-factor in the SM wire that allows to have the magnetic field values affecting the electronic states in the wire and barely affecting the ones in the superconducting cover. Note that omitting the orbital effects we cannot describe possible Little-Parks effect arising in the wires fully covered by the $\mathrm{S}$ shell $[27,28]$.

Neglecting the order parameter inhomogeneity in the shell for $d_{s} \ll \xi_{s}$, we derive the following system of Gor'kov equations written in the frequency-momentum representation (see Supporting Information File 1 for the details of the derivation):

$$
\left(i \omega_{n}-\varepsilon_{s} \check{\tau}_{z}+\check{\Delta}-\check{\Sigma}_{s}\right) \check{G}_{s}=\check{1}
$$

$$
\left(i \omega_{n}-\varepsilon_{w} \check{\tau}_{z}-\alpha p_{y} \hat{\sigma}_{x}-h \hat{\sigma}_{y}-\check{\Sigma}_{w}\right) \check{G}_{w}=\check{1}
$$

where $\omega_{n}=2 \pi T(n+1 / 2)$ is the Matsubara frequency, $T$ is the temperature, $p_{y}$ is the momentum along the wire, $\check{\tau}_{m}$ ( $m=x, y$, $z$ ) are the Pauli matrices acting in the Nambu space, $\check{\Delta}=\left(\hat{\Delta} \check{\tau}_{+}+\hat{\Delta}^{\dagger} \breve{\tau}_{-}\right), \hat{\Delta}=\Delta_{s}\left(i \hat{\sigma}_{y}\right), \Delta_{s}$ is the superconducting order parameter, which we assume to be constant in space and realvalued, $\check{\tau}_{ \pm}=\left(\check{\tau}_{x} \pm i \check{\tau}_{y}\right) / 2$, and $\varepsilon_{w}=p_{y}^{2} / 2 m_{w}-\mu_{w}$. The tunneling self-energy parts are given by the following expression:

$$
\check{\Sigma}_{s(w)}=\Gamma_{w(s)} \check{\tau}_{z} \check{g}_{w(s)} \check{\tau}_{z}
$$

where $\Gamma_{s}=t^{2} \ell_{c} R_{w} m_{s} \varphi_{0} / 2$ and $\Gamma_{w}=t^{2} \ell_{c} / 2 v_{0}$. The functions $\check{g}_{s(w)}$ are the quasiclassical Green's functions:

$$
\check{g}_{s}=\frac{1}{\pi} \int \mathrm{d} \varepsilon_{s} \check{G}_{s}\left(\omega_{n}, \varepsilon_{s}\right),
$$




$$
\check{g}_{w}=\frac{\cup_{0}}{\pi} \int \mathrm{d} p_{y} \check{G}_{w}\left(\omega_{n}, p_{y}\right)
$$

The precise definitions of the Green's functions $\check{G}_{w, s}$ of the wire and of the shell, respectively, together with the derivation of Equation 6 and Equation 7 are given in Supporting Information File 1.

Note that we neglect here the possible dependence of these quasiclassical Green's functions on the coordinate along the wire. That is, we assume the limit of an infinitely long wire without edge effects. The velocity $v_{0}$ is introduced just for the purpose of unification of dimensionality of the tunneling rates $\Gamma_{w}$ and $\Gamma_{s}$ and does not appear in the product $\Gamma_{w} \check{g}_{w}$ that enters the measurable quantities. One can choose this velocity, e.g., as $v_{0}=\sqrt{2 \mu_{w} / m_{w}}$ so that the rate $\Gamma_{w}$ includes the divergent DOS in the $1 \mathrm{D}$ wire.

Tunneling rates for the quasiparticles from the shell into the wire, $\Gamma_{w}$, and from the wire into the shell, $\Gamma_{s}$, can be expressed in terms of the normal-state tunnel resistance $\mathcal{R}$ in the following manner [20]:

$$
\begin{gathered}
\Gamma_{w}=\frac{1}{4 \pi \mathcal{R} S G_{0} v_{s}} \propto \mu_{s} \frac{\mathcal{R}_{0}}{\mathcal{R}} \frac{k_{F w}}{k_{F s}} \frac{1}{\left(k_{F s} R_{w}\right)}, \\
\Gamma_{s}=\frac{1}{4 \pi \mathcal{R} \ell_{w} G_{0} v_{w}} \propto \mu_{w} \frac{\mathcal{R}_{0}}{\mathcal{R}},
\end{gathered}
$$

where $S=2 \pi R_{w} \ell_{w}$ is the contact area, $\ell_{w}$ is the wire length, $G_{0}=e^{2} / \pi$ is the conductance quantum, $v_{S}=m_{S} / 2 \pi$ and $v_{w}=\left(2 m_{w} / \mu_{w}\right)^{1 / 2}$ are the normal DOS in the shell and in the wire, respectively, $\mathcal{R}_{0}=\left(N G_{0}\right)^{-1}, N=k_{F w} \ell_{w}$, and $k_{F s(w)}$ is the Fermi momentum in the shell (wire). The expressions for the tunneling rates can be conveniently written through the numbers of transverse modes in the superconducting shell $\left(N_{s} \approx k_{F_{s}}^{2} d_{s} R_{w} \varphi_{0}\right)$ and in the wire $\left(N_{w}\right)$ :

$$
\begin{aligned}
& \Gamma_{s} \approx t^{2} \ell_{c} \frac{N_{s}}{\mathrm{v}_{F_{s}}}, \\
& \Gamma_{w} \approx t^{2} \ell_{c} \frac{N_{w}}{\mathrm{v}_{0}},
\end{aligned}
$$

where $v_{F_{s}}=k_{F_{s}} / m_{s}$. Here we use the simplest generalization [17] of the expression for $\Gamma_{w}$ for the case of an arbitrary number of transverse modes in the nanowire assuming also the value $1 / v_{0}$ to be averaged over these modes. The resulting ratio of the tunneling rates takes the form:

$$
\frac{\Gamma_{w}}{\Gamma_{s}} \approx \frac{N_{w}}{N_{s}} \frac{v_{F_{s}}}{v_{0}}
$$

Due to the growth of $N_{S}$ with the shell thinkness $d_{S}$ in the multimode regime of the superconductor this ratio may become rather small weakening the inverse proximity effect (the details of experimental relevance are considered in the next section).

Equation 6 and Equation 7 must be solved together with the self-consistency equation for the superconducting gap function:

$$
\Delta_{s}^{*}=\frac{\lambda \pi T}{2} \sum_{\omega_{n}} \operatorname{Tr}\left[\left(\check{g}_{s}\right)_{21}\left(i \hat{\sigma}_{y}\right)\right] \text {, }
$$

where $\lambda$ is the dimensionless pairing constant and the trace is taken over the spin indices. The next section is devoted to the perturbative solution of the Gor'kov equations (Equation 6 and Equation 7) and the self-consistency equation (Equation 16) in the gap potential which allows one to find the critical temperature of superconducting transition as a function of magnetic field and materials parameters.

\section{Results and Discussion}

Considering the perturbation theory in the superconducting gap function $\Delta_{S}$ it is natural to start with the equations for the normal Green's functions

$$
\begin{gathered}
\left(i \omega_{n}-\varepsilon_{s}-\Gamma_{w} \hat{g}_{w}\right) \hat{G}_{s}=\hat{1} \\
\left(i \omega_{n}+\varepsilon_{s}-\Gamma_{w} \hat{\bar{g}}_{w}\right) \hat{\bar{G}}_{s}=\hat{1} \\
\left(i \omega_{n}-\varepsilon_{w}-\alpha p_{y} \hat{\sigma}_{x}-h \hat{\sigma}_{y}-\Gamma_{s} \hat{g}_{s}\right) \hat{G}_{w}=\hat{1}, \\
\left(i \omega_{n}+\varepsilon_{w}-\alpha p_{y} \hat{\sigma}_{x}-h \hat{\sigma}_{y}-\Gamma_{s} \hat{\bar{g}}_{s}\right) \hat{\bar{G}}_{w}=\hat{1},
\end{gathered}
$$

which give us the zero-order solution of the Gor'kov equations

$$
\begin{gathered}
\hat{G}_{s}=\frac{i \omega_{n}-\varepsilon_{s}-\Gamma_{w} g_{w 0}+\Gamma_{w} \mathbf{g}_{w} \hat{\boldsymbol{\sigma}}}{\left(i \omega_{n}-\varepsilon_{s}-\Gamma_{w} g_{w 0}\right)^{2}-\left(\Gamma_{w} \mathbf{g}_{w}\right)^{2}}, \\
\hat{G}_{w}=\frac{i \omega_{n}-\varepsilon_{w}-\Gamma_{s} g_{s 0}+\mathbf{U}_{w} \hat{\boldsymbol{\sigma}}}{\left(i \omega_{n}-\varepsilon_{w}-\Gamma_{s} g_{s 0}\right)^{2}-\mathbf{U}_{w}^{2}} .
\end{gathered}
$$


Here, $\mathbf{U}_{w}=\left[\left(\alpha p_{y}+\Gamma_{s} g_{s x}\right),\left(h+\Gamma_{s} g_{s y}\right), \Gamma_{s} g_{s z}\right]$ and the quasiclassical Green's functions are written in the spin form

$$
\hat{g}_{k}=g_{k 0}+\mathbf{g}_{k} \hat{\boldsymbol{\sigma}}
$$

with $k=s(w)$ for the shell (wire). The solutions for spin matrix functions $\hat{\bar{G}}_{k}$ are given by Equation 21 and Equation 22 with the replacements $\varepsilon_{k} \rightarrow-\varepsilon_{k}$ and $\hat{g}_{k} \rightarrow \hat{\bar{g}}_{k}$.

According to the definitions for the quasiclassical Green's functions (Equation 9 and Equation 10) and due to a specific spin structure of the Zeeman term and spin-orbit coupling term in Equation 17-Equation 20, one can easily get that only $g_{k 0}$ and $g_{k y}$ are nonzero. It is convenient to rewrite the normal Green's function in the wire as a sum of singular contributions $G_{w}^{p^{ \pm}}$:

$$
\begin{aligned}
\hat{G}_{w}= & \frac{\left(\varepsilon_{s o}+\varepsilon_{0}\right) G_{w}^{p+}-\left(\varepsilon_{s o}-\varepsilon_{0}\right) G_{w}^{p-}}{2 \varepsilon_{0}} \\
& -\frac{\left(G_{w}^{p+}-G_{w}^{p-}\right)}{2 \varepsilon_{0}}\left(\alpha p_{y} \hat{\sigma}_{x}+h \hat{\sigma}_{y}\right), \\
\hat{\bar{G}}_{w}= & \frac{-\left(\varepsilon_{s o}-\varepsilon_{0}^{*}\right) \bar{G}_{w}^{p+}+\left(\varepsilon_{s o}+\varepsilon_{0}^{*}\right) \bar{G}_{w}^{p-}}{2 \varepsilon_{0}^{*}} \\
& -\frac{\left(\bar{G}_{w}^{p+}-\bar{G}_{w}^{p-}\right)}{2 \varepsilon_{0}^{*}}\left(\alpha p_{y} \hat{\sigma}_{x}+h \hat{\sigma}_{y}\right),
\end{aligned}
$$

where

$$
\begin{aligned}
G_{w}^{p \pm} & =\left(i \bar{\omega}_{n}-\varepsilon_{w}+\varepsilon_{s o} \pm \varepsilon_{0}\right)^{-1}, \\
\bar{G}_{w}^{p \pm} & =-\left(G_{w}^{p \mp}\right)^{*}, \\
\varepsilon_{0} & =\sqrt{2 \varepsilon_{s o}\left(\mu_{w}+i \bar{\omega}_{n}\right)+\varepsilon_{s o}^{2}+h^{2}}, \\
\varepsilon_{s o} & =m_{w} \alpha^{2} \text { and } \\
\bar{\omega}_{n} & =\omega_{n}+\Gamma_{s} \operatorname{sign}\left(\omega_{n}\right) .
\end{aligned}
$$

The equations for the anomalous Green's functions read:

$$
\left(i \omega_{n}+\varepsilon_{s}-\Gamma_{w} \hat{\bar{g}}_{w}\right) \hat{F}_{s}^{\dagger}=-\left(\hat{\Delta}^{\dagger}+\Gamma_{w} \hat{f}_{w}^{\dagger}\right) \hat{G}_{s}
$$

$$
\left(i \omega_{n}+\varepsilon_{w}-\alpha p_{y} \hat{\sigma}_{x}-h \hat{\sigma}_{y}-\Gamma_{s} \hat{\bar{g}}_{s}\right) \hat{F}_{w}^{\dagger}=-\Gamma_{s} \hat{f}_{s}^{\dagger} \hat{G}_{w}
$$

and give the solution for the anomalous Green's functions $\hat{F}_{k}^{\dagger}$ within the first-order perturbation theory in the superconducting gap:

$$
\hat{F}_{s}^{\dagger}=-\hat{\bar{G}}_{s}\left(\hat{\Delta}^{\dagger}+\Gamma_{w} \hat{f}_{w}^{\dagger}\right) \hat{G}_{s}
$$

$$
\hat{F}_{w}^{\dagger}=-\hat{\bar{G}}_{w} \Gamma_{s} \hat{f}_{s}^{\dagger} \hat{G}_{w}
$$

Introducing a general presentation for the components of the quasiclassical anomalous Green's functions

$$
\hat{f}_{k}^{\dagger}=-i \hat{\sigma}_{y}\left(f_{k 0}^{\dagger}+\mathbf{f}_{k}^{\dagger} \hat{\boldsymbol{\sigma}}\right)
$$

we get the set of equations in Equation 31 for them with $f_{s x}^{\dagger}=f_{s z}^{\dagger}=0$.

The solutions of Equation 31 take the form given in Equation 32 and Equation 33.

We use the following notations:

$$
\begin{aligned}
I_{v \eta}^{w} & =\int \frac{\mathrm{d} p_{y}}{\pi} \bar{G}_{w}^{p v} G_{w}^{p \eta} \\
& =\frac{\bar{g}_{w}^{p v}+g_{w}^{p \eta}}{2 i \bar{\omega}_{n}+v \varepsilon_{0}^{*}+\eta \varepsilon_{0}}
\end{aligned}
$$




$$
\begin{aligned}
& \begin{array}{c}
f_{s y}^{\dagger}=-\Delta_{s} \overline{[1-} \\
\frac{p_{y}^{2}}{2 m_{w}} \bar{G}_{w}^{p v} G_{w}^{p \eta}
\end{array} \\
& =\frac{\bar{g}_{w}^{p v}\left(-i \bar{\omega}_{n}-v \varepsilon_{0}^{*}+\mu_{w}+\varepsilon_{s o}\right)}{\left(2 i \bar{\omega}_{n}+\eta \varepsilon_{0}+v \varepsilon_{0}^{*}\right)} \\
& \frac{+g_{w}^{p \eta}\left(i \bar{\omega}_{n}+\eta \varepsilon_{0}+\mu_{w}+\varepsilon_{s o}\right)}{\left(2 i \bar{\omega}_{n}+\eta \varepsilon_{0}+v \varepsilon_{0}^{*}\right)} \\
& I_{w x}=\sum_{v, \eta= \pm 1} v \eta \frac{\varepsilon_{s o} I_{v \eta}^{w x}}{2\left|\varepsilon_{0}\right|^{2}} \\
& I_{w 0}=\sum_{v, \eta= \pm 1}\left[\left(\varepsilon_{0}^{*}-v \varepsilon_{s o}\right)\left(\varepsilon_{0}+\eta \varepsilon_{s o}\right)+h^{2} v \eta\right] \frac{I_{v \eta}^{w}}{4\left|\varepsilon_{0}\right|^{2}} \\
& I_{w y}=\sum_{v, \eta= \pm 1}\left(\eta \varepsilon_{0}^{*}+v \varepsilon_{0}\right) \frac{(-h) I_{v \eta}^{w}}{4\left|\varepsilon_{0}\right|^{2}}
\end{aligned}
$$

In addition, $v, \eta= \pm 1$. The expressions for the integrals involving the products of the normal Green's functions in the shell can be written as follows:

$$
\begin{gathered}
I_{s 0}=I_{s+}+I_{s-} \\
I_{s y}=I_{s+}-I_{s-} \\
I_{s \eta}=\int \frac{\mathrm{d} \varepsilon_{s}}{(2 \pi)} \bar{G}_{s \eta} G_{s \eta}=\frac{1}{2} \frac{\left(\bar{g}_{s \eta}+g_{s \eta}\right)}{\left[2 i \omega_{n}-\Gamma_{w}\left(\bar{g}_{w \eta}+g_{w \eta}\right)\right]}
\end{gathered}
$$

In the definitions in Equation 34 and Equation 35 and Equation 39-Equation 41, we have introduced the following functions:

$$
\begin{gathered}
\bar{g}_{s \eta}=g_{s \eta}=g_{s 0}=-i \operatorname{sign}\left(\omega_{n}\right), \quad g_{s y}=0, \\
g_{w}^{p \eta}=-2 i \sqrt{\frac{m_{w}}{2}} \frac{\operatorname{sign}\left(\bar{\omega}_{n}+\eta \varepsilon_{I}\right)}{\sqrt{\eta \varepsilon_{0}+\mu_{w}+\varepsilon_{s o}+i \bar{\omega}_{n}}} .
\end{gathered}
$$

Here, $g_{k \eta}=\left(g_{k 0}+\eta g_{k y}\right), \bar{g}_{w}^{p \eta}=-\left[g_{w}^{p(-\eta)}\right]^{*}$, and $\varepsilon_{I}=\operatorname{Im}\left(\varepsilon_{0}\right)$. Finally, we explicitly show the expressions for the normal Green's functions in the wire:

$$
\begin{gathered}
g_{w 0}=-i \sqrt{\frac{m_{w}}{2}} \sum_{\eta= \pm 1} \frac{\left(\varepsilon_{0}+\eta \varepsilon_{s o}\right) \operatorname{sign}\left(\bar{\omega}_{n}+\eta \varepsilon_{I}\right)}{\varepsilon_{0} \sqrt{\eta \varepsilon_{0}+\mu_{w}+\varepsilon_{s o}+i \bar{\omega}_{n}}} \\
g_{w y}=i h \sqrt{\frac{m_{w}}{2}} \sum_{\eta= \pm 1} \frac{\eta \operatorname{sign}\left(\bar{\omega}_{n}+\eta \varepsilon_{I}\right)}{\varepsilon_{0} \sqrt{\eta \varepsilon_{0}+\mu_{w}+\varepsilon_{s o}+i \bar{\omega}_{n}}}
\end{gathered}
$$

Note that in the absence of spin-orbit coupling, zero magnetic field and for energy-independent DOS in the wire the selfconsistency equation formally coincides with the one obtained in the seminal work by McMillan [17].

Turning now to the case of nonzero Zeeman energy and spin-orbit coupling we use a numerical approach to analyze the solution of the self-consistency Equation 16 with the Equation 32 and Equation 33 for the anomalous Green function. Typical dependencies of the critical superconducting temperature on the magnetic field and chemical potential $\mu_{w}$ are shown in Figure 2. Note that here we choose the strength of the spin-orbit coupling consistent with the properties of InAs [22]: $\varepsilon_{s o}=m_{w} \alpha^{2}=52 \mu \mathrm{eV}$, which corresponds to approximately $600 \mathrm{mK}$. Taking the critical temperature of $\mathrm{Al} T_{c 0} \approx 1.3 \mathrm{~K}$, we find $\varepsilon_{s o}=m_{w} \alpha^{2}=0.46 T_{c 0}$.

The color plots in Figure 2 show the critical temperature $T_{c}$ both in topologically trivial $\left(\left|\mu_{w}\right|>h\right)$ and nontrivial $\left(\left|\mu_{w}\right|<h\right)$ regimes. The border lines $\mu_{w}= \pm h$ (shown by white dashed lines) coincide with the locations of van Hove singularities in the SM nanowire. One can clearly see that the suppression of the critical temperature appears to be the strongest close to these lines. The magnetic field dependence of $T_{c}$ appears to be drastically different in topologically trivial and nontrivial regimes. Indeed, in the nontopological regime the critical temperature decays as we increase the magnetic field due to a standard paramagnetic effect. In contast, in the topologically nontrivial regime $T_{c}$ increases (with or without initial decay at small fields). This increase in the critical temperature originates from the reduction of the proximity effect due to almost pure spin polarization of quasiparticles in the wire. The above mentioned increase in the critical temperature is limited from above by either orbital or intrinsic paramagnetic effects in the $\mathrm{S}$ shell and 

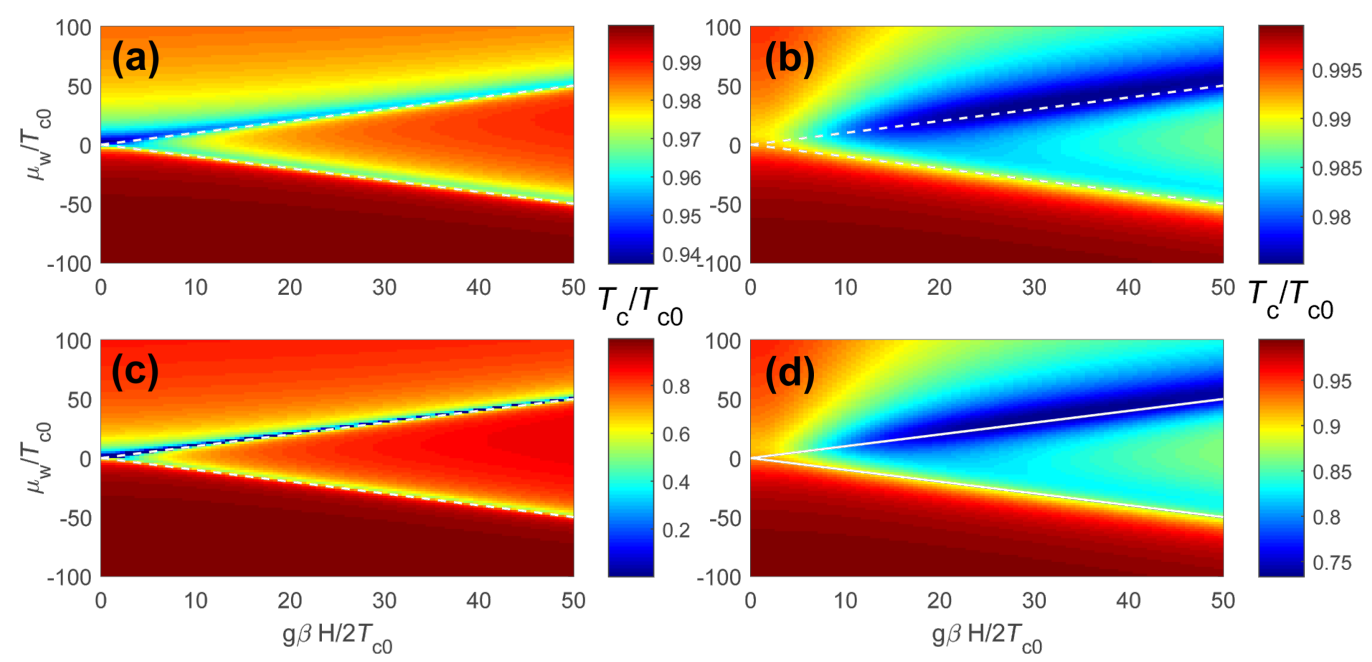

Figure 2: Color plot of the critical temperature of the system as a function of the chemical potential $\mu_{w}$ and the Zeeman energy $h=g \beta H / 2$ for $\varepsilon_{s o}=m_{w} a^{2}=0.46 T_{c 0}$ and several values of $\Gamma_{s}=t^{2} \ell_{c} R_{w} m_{s} \varphi_{0} / 2$ and $\Gamma_{w}=t^{2} \ell_{c} / 2 \mathrm{U}_{0}$ with $\mathrm{u}_{0}=\sqrt{2 T_{c 0} / m_{w}}$. In panels (a) and (b) $\Gamma_{w}=0.1 T_{c 0}$, while in panels (c) and (d) we take $\Gamma_{w}=T_{c 0}$. In panels (a) and (c) $\Gamma_{s}=0.1 T_{c 0}$, in panels (b) and (d) $\Gamma_{s}=10 T_{c 0}$. In all panels the white dashed lines denote the boundaries between nontopological and topological regimes $\mu_{w}= \pm h$.

continues up to the upper critical field in the superconductor. One can see that the scattering rates $\Gamma_{w}$ and $\Gamma_{s}$ have a strong quantitative effect on the above physical picture because of smearing and shifting of the peculiarities of the DOS and the resulting smoothing of $T_{c}$ variations. The nonmonotonic behavior of $T_{c}$ is illustrated by the plots in Figure 3. Using the above expressions in Equation 13-Equation 15 for the tunneling rates, we estimate the ratio of mode numbers as $N_{w} / N_{S} \approx 10^{-5}-10^{-4}$ for typical Majorana nanowires [11-16]. Taking into account the decrease of the $v_{0}$ value close to the van Hove singularity $\left(v_{F_{s}} / v_{0} \approx 10^{2}-10^{3}\right)$, we get $\Gamma_{w} / \Gamma_{s} \approx 10^{-3}-10^{-1}$. Assuming strong coupling between the nanowire and superconducting shell with $\Gamma_{s} \geq T_{c 0}$, we get $\Gamma_{w} \approx\left(10^{-3}-10^{-1}\right) T_{c 0}$. Note that under realistic experimental conditions the number of modes in the wire $\left(N_{w}\right)$ can increase due to the formation of the accumu- lation layer near the superconductor-semiconductor interface [29-31]. However, the increase of the shell thickness $d_{s}$ may weaken the effect in the multimode regime of the superconductor. Overall, such estimate allows us to expect that the consequences of the inverse proximity effect analyzed in our paper can be observed experimentally.

It is worth noting that the $T_{c}(h)$ plot in the Figure 3 a clearly demonstrates the appearance of $h$ regions where the linearized self-consistency equation has three solutions instead of one. In other words, there can exist three critical temperatures corresponding to a given magnetic field. This is evidence for the fact that although the superconducting shell has a small $g$-factor, the indirectly superconducting region is affected by effective Zeeman field through tunneling. The presence of several solu-
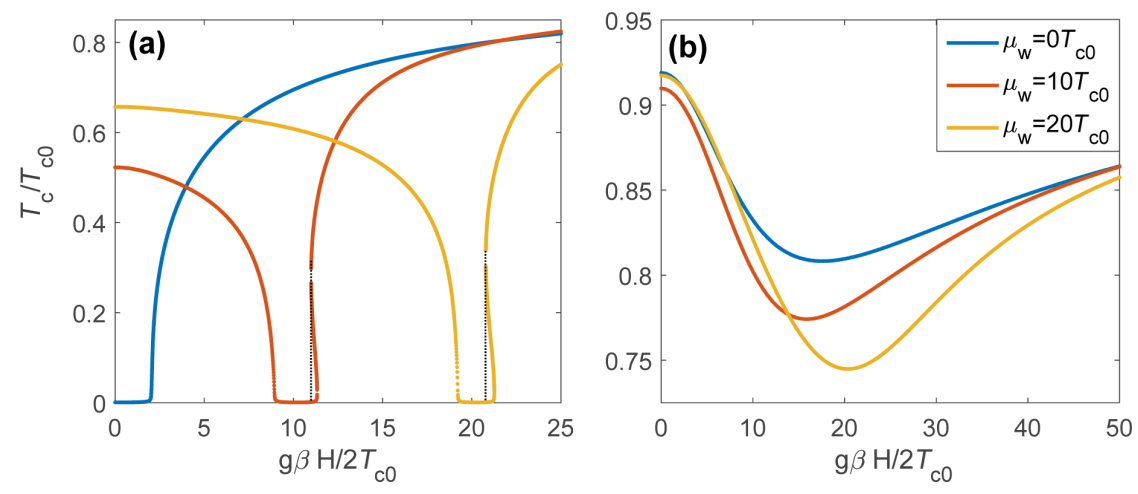

Figure 3: The critical temperature of the system as a function of the Zeeman field $h$ for different values of the chemical potential in the wire $\mu_{w}$ (shown in the legend). Here, $\varepsilon_{s o}=0.46 T_{c 0}$ and $\Gamma_{w}=T_{c 0}$. (a) $\Gamma_{s}=0.1 T_{c 0}$ and (b) $\Gamma_{s}=10 T_{c 0}$. 
tions for $T_{c}$ is typical for the standard paramagnetic effect in superconductors and usually this behavior results in the FFLO instability of the homogeneous solution for the gap function [32]. To verify this scenario in our system we have solved a self-consistency equation for the modulated order parameter $\Delta_{s} \propto e^{i q y}$ and found that the regions with several solutions for $T_{c}$ for the homogeneous gap can, indeed, host an energetically more favorable inhomogeneous FFLO gap function. The critical temperature $T_{c}(q)$ for different $q$ values can be seen in Figure 4. As we increase the $h$ value from $h=10.8$ to $h=11.05$ the $q$ value corresponding to the maximal $T_{c}$ changes from $k_{F s} q / m_{s}=T_{c 0}$ to $k_{F s} q / m_{s}=0.44 T_{c 0}$. It is important to note that as we solve the linearized equation for the superconducting gap, we find, of course, only the critical temperatures corresponding to the second-order phase transitions. Changing the period of the gap modulation of the FFLO-type we also find only the temperatures corresponding to the second-order phase transition. The physical picture can become more complicated if one takes into account possible first-order transitions corresponding to the interplay between different local minima of the thermodynamic potential in the nonlinear regime. However, the solution of nonlinear gap equations is beyond the scope of the current work and needs further investigations. Note also that the possible FFLO phase appears on either side of topological transition ( $h^{2}=\mu_{w}^{2}+\Delta_{\text {ind }}^{2}$ ) depending on the sign of the chemical potential $\mu_{w}$. Indeed, in general the temperature as a formal solution of the self-consistency Equation 16 is not a single-valued function of the magnetic field in the regions $h \geq \pm \mu_{w}$ slightly above the positions of van Hove singularities, being inside the topological (trivial) regime for the upper (lower) sign. In experimentally feasible cases of considered $\Gamma_{s, w}$ (Figure 2) the upper singularity at $\mu_{\mathrm{w}}=h$ is more pronounced (Figure 3). Additionally, an accurate analysis of the FFLO state should include careful

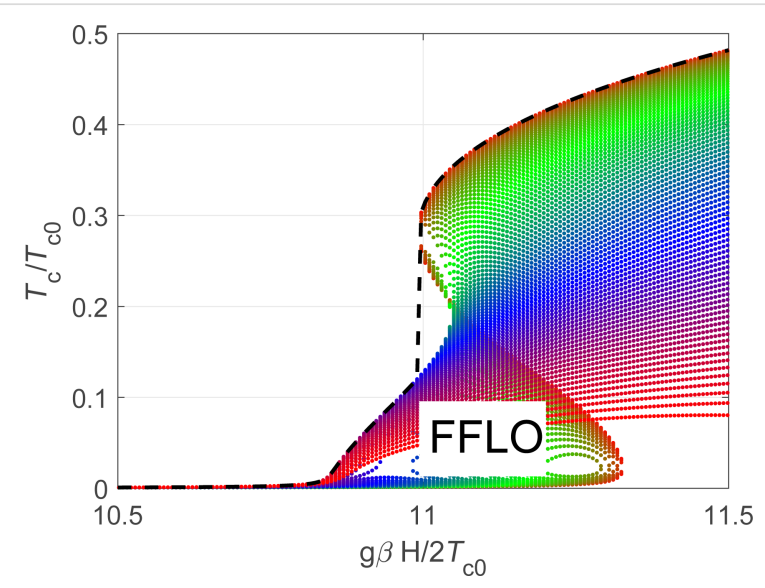

Figure 4: Critical temperature of the system as a function of the Zeeman field $h$ for $\varepsilon_{s o}=0.46 T_{c 0}, \Gamma_{s} \rightarrow 0$ and $\Gamma_{w}=T_{c 0}$ for the superconducting states with different modulation vectors $q$ ranging from $q=0.44 m_{s} T_{c O} / k_{F s}$ at $h=11.05 T_{c O}$ to $q=m_{s} T_{c O} / k_{F s}$ at $h=10.8 T_{c 0}$. consideration of the modulation of the superconducting order parameter both along the wire and in the azimuthal direction $[27,28]$.

Before we conclude, we discuss briefly the influence of the inverse proximity effect on the effective induced gap operator $\Delta_{\text {top }}$ in the topological regime, $h^{2}>\mu_{w}^{2}+\Delta_{\text {ind }}^{2}$, which is of crucial importance for topological superconducting electronics and topologically protected fault-tolerant quantum computing. In our estimates we take the standard limit of $\mu_{w}=0$ for the sake of simplicity. First, the increase of $\Gamma_{w}$ reduces the parameter range of the topological insulator regime $\alpha p \gg h$ as the magnetic field should well exceed $\Gamma_{w}$ to avoid the suppression of the critical temperature due to the van Hove singularities (see Figure 2a,c for small $\Gamma_{s}$ values). As soon as $\Gamma_{w}$ becomes comparable with $\alpha p$ with the typical quasiparticle momentum $p \simeq \sqrt{2 m_{w} h}$ this regime completely disappears. Further increase of the scattering rate should suppress the gap $\Delta_{\text {top }} \propto \alpha p \Delta_{\text {ind }} / h$ in the Kitaev limit. Indeed, for $\Gamma_{w}>m_{w} \alpha^{2}=\varepsilon_{s o}$ its value is limited from above by the quantity $\Delta_{\text {top }} \approx \Delta_{\text {ind }}\left(\varepsilon_{s o} / \Gamma_{w}\right)^{1 / 2}<\Delta_{\text {ind }}$. Such decrease in the attainable induced gap values imposes more strict conditions on working temperatures for Majorana-based devices, due to quasiparticle poisoning as the residual quasiparticle density is exponentially sensitive to the gap values [33-36]. Of course, at large values of $\Gamma_{s}$ (see Figure 2b,d) the van Hove singularities are smeared and the critical temperature (together with the gap value) is suppressed only partially. However, even the partial suppression up to tens of percents may drastically increase the effect of quasiparticle poisoning mentioned above.

\section{Conclusion}

We have studied the distinctive features of the inverse proximity effect arising in the presence of a large Zeeman energy and strong spin-orbit coupling in the hybrid systems consisting of the SM nanowires covered by thin superconducting films. Assuming a strong difference in $g$-factors between the wire and superconducting metal we find the range of parameters and fields corresponding to the FFLO instability and the regime of reentrant superconductivity. We focus on the topologically nontrivial regime of relatively large magnetic fields and analyze consequences of the inverse proximity effect on the quasiparticle poisoning in Majorana-based devices.

\section{Supporting Information}

\section{Supporting Information File 1}

Derivation of the model Equation 6 and Equation 7.

[https://www.beilstein-journals.org/bjnano/content/

supplementary/2190-4286-9-109-S1.pdf] 


\section{Acknowledgements}

We are pleased to thank J. P. Pekola for valuable and stimulating discussions. This work has been supported in part by the Russian Foundation for Basic Research Grant Nos. 17-5212044, 18-02-00390 (A. A. K.), German Research Foundation (DFG) Grant No. KH 425/1-1 (I. M. K.), by the Russian Science Foundation, Grant No. 17-12-01383 (A. S. M.), Foundation for the advancement of theoretical physics "BASIS", and the Academy of Finland Grant No. 298451.

\section{ORCID ${ }^{\circledR}$ iDs}

Ivan M. Khaymovich - https://orcid.org/0000-0003-2160-5984

\section{References}

1. Kitaev, A. Yu. Phys.-Usp. 2001, 44, 131. doi:10.1070/1063-7869/44/10S/S29

2. Alicea, J. Phys. Rev. B 2010, 81, 125318. doi:10.1103/PhysRevB.81.125318

3. Lutchyn, R. M.; Sau, J. D.; Das Sarma, S. Phys. Rev. Lett. 2010, 105, 077001. doi:10.1103/PhysRevLett.105.077001

4. Oreg, Y.; Refael, G.; von Oppen, F. Phys. Rev. Lett. 2010, 105, 177002. doi:10.1103/PhysRevLett.105.177002

5. Nayak, C.; Simon, S. H.; Stern, A.; Freedman, M.; Das Sarma, S. Rev. Mod. Phys. 2008, 80, 1083-1159. doi:10.1103/RevModPhys.80.1083

6. Alicea, J.; Oreg, Y.; Refael, G.; von Oppen, F.; Fisher, M. P. A. Nat. Phys. 2011, 7, 412. doi:10.1038/nphys1915

7. Aasen, D.; Hell, M.; Mishmash, R. V.; Higginbotham, A.; Danon, J.; Leijnse, M.; Jespersen, T. S.; Folk, J. A.; Marcus, C. M.; Flensberg, K.; Alicea, J. Phys. Rev. X 2016, 6, 031016. doi:10.1103/PhysRevX.6.031016

8. Alicea, J. Rep. Prog. Phys. 2012, 75, 076501. doi:10.1088/0034-4885/75/7/076501

9. Elliott, S. R.; Franz, M. Rev. Mod. Phys. 2015, 87, 137-163. doi:10.1103/RevModPhys.87.137

10. Aguado, R. Riv. Nuovo Cimento Soc. Ital. Fis. 2017, 40, 523. doi:10.1393/ncr/i2017-10141-9

11. Mourik, V.; Zuo, K.; Frolov, S. M.; Plissard, S. R.; Bakkers, E. P. A. M.; Kouwenhoven, L. P. Science 2012, 336, 1003-1007. doi:10.1126/science. 1222360

12. Chang, W.; Albrecht, S. M.; Jespersen, T. S.; Kuemmeth, F.; Krogstrup, P.; Nygård, J.; Marcus, C. M. Nat. Nanotechnol. 2015, 10, 232. doi:10.1038/nnano.2014.306

13. Higginbotham, A. P.; Albrecht, S. M.; Kiršanskas, G.; Chang, W.; Kuemmeth, F.; Krogstrup, P.; Jespersen, T. S.; Nygård, J.; Flensberg, K.; Marcus, C. M. Nat. Phys. 2015, 11, 1017. doi:10.1038/nphys3461

14. Krogstrup, P.; Ziino, N. L. B.; Chang, W.; Albrecht, S. M.; Madsen, M. H.; Johnson, E.; Nygård, J.; Marcus, C. M.; Jespersen, T. S. Nat. Mater. 2015, 14, 400. doi:10.1038/nmat4176

15. Albrecht, S. M.; Higginbotham, A. P.; Madsen, M.; Kuemmeth, F.; Jespersen, T. S.; Nygård, J.; Krogstrup, P.; Marcus, C. M. Nature 2016, 531, 206. doi:10.1038/nature 17162
16. Zhang, H.; Gül, Ö.; Conesa-Boj, S.; Nowak, M. P.; Wimmer, M.; Zuo, K.; Mourik, V.; de Vries, F. K.; van Veen, J.; de Moor, M. W. A.; Bommer, J. D. S.; van Woerkom, D. J.; Car, D.; Plissard, S. R.; Bakkers, E. P. A. M.; Quintero-Pérez, M.; Cassidy, M. C.; Koelling, S.; Goswami, S.; Watanabe, K.; Taniguchi, T.; Kouwenhoven, L. P. Nat. Commun. 2017, 8, 16025. doi:10.1038/ncomms 16025

17. McMillan, W. L. Phys. Rev. 1968, 175, 537-542. doi:10.1103/PhysRev.175.537

18. Sau, J. D.; Tewari, S.; Lutchyn, R. M.; Stanescu, T. D.; Das Sarma, S. Phys. Rev. B 2010, 82, 214509. doi:10.1103/PhysRevB.82.214509

19. Kopnin, N. B.; Melnikov, A. S. Phys. Rev. B 2011, 84, 064524. doi:10.1103/PhysRevB.84.064524

20. Kopnin, N. B.; Khaymovich, I. M.; Mel'nikov, A. S. Phys. Rev. Lett. 2013, 110, 027003. doi:10.1103/PhysRevLett.110.027003

21. Kopnin, N. B.; Khaymovich, I. M.; Mel'nikov, A. S. J. Exp. Theor. Phys. 2013, 117, 418. doi:10.1134/S1063776113110113

22. Stanescu, T. D.; Lutchyn, R. M.; Das Sarma, S. Phys. Rev. B 2011, 84, 144522. doi:10.1103/PhysRevB.84.144522

23. Reeg, C.; Loss, D.; Klinovaja, J. Phys. Rev. B 2017, 96, 125426. doi:10.1103/PhysRevB.96.125426

24. Reeg, C.; Loss, D.; Klinovaja, J. arXiv 2018, No. 1801.06509.

25. Larkin, A. I.; Ovchinnikov, Yu. N. Sov. Phys. - JETP 1965, 20, 762.

26. Fulde, P.; Ferrell, R. A. Phys. Rev. 1964, 135, A550. doi:10.1103/PhysRev.135.A550

27. Samokhvalov, A. V.; Mel'nikov, A. S.; Ader, J.-P.; Buzdin, A. I. Phys. Rev. B 2009, 79, 174502. doi:10.1103/PhysRevB.79.174502

28. Samokhvalov, A. V. J. Exp. Theor. Phys. 2017, 125, 298-309. doi:10.1134/S106377611707010X

29. Antipov, A. E.; Bargerbos, A.; Winkler, G. W.; Bauer, B.; Rossi, E.; Lutchyn, R. M. arXiv 2018, 1801.02616.

30. Mikkelsen, A. E. G.; Kotetes, P.; Krogstrup, P.; Flensberg, K. arXiv 2018, 1801.03439.

31. Woods, B. D.; Stanescu, T. D.; Das Sarma, S. arXiv 2018, 1801.02630.

32. Saint-James, D.; Sarma, G.; Thomas, E. Type /I Superconductivity; Pergamon Press: Oxford, United Kingdom, 1969.

33. Saira, O.-P.; Kemppinen, A.; Maisi, V. F.; Pekola, J. P. Phys. Rev. B 2012, 85, 012504. doi:10.1103/PhysRevB.85.012504

34. Knowles, H. S.; Maisi, V. F.; Pekola, J. P. Appl. Phys. Lett. 2012, 100, 262601. doi:10.1063/1.4730407

35. Maisi, V. F.; Lotkhov, S. V.; Kemppinen, A.; Heimes, A.; Muhonen, J. T.; Pekola, J. P. Phys. Rev. Lett. 2013, 111, 147001. doi:10.1103/PhysRevLett.111.147001

36. van Woerkom, D. J.; Geresdi, A.; Kouwenhoven, L. P. Nat. Phys. 2015, 11, 547. doi:10.1038/nphys3342 


\section{License and Terms}

This is an Open Access article under the terms of the Creative Commons Attribution License

(http://creativecommons.org/licenses/by/4.0), which permits unrestricted use, distribution, and reproduction in any medium, provided the original work is properly cited.

The license is subject to the Beilstein Journal of Nanotechnology terms and conditions:

(https://www.beilstein-journals.org/bjnano)

The definitive version of this article is the electronic one which can be found at:

doi:10.3762/bjnano.9.109 